\title{
WSNs and on-Board Visual Fuzzy Servoing on Blimp Robot for Tracking Purposes
}

\author{
Rami Al-Jarrah ${ }^{1}$, Mohammad A. Al-Jarrah², Hubert Roth ${ }^{1}$ \\ ${ }^{1}$ Automatic Control Engineering Department, Siegen University, Siegen, Germany. \\ 2 The Electrical Engineering Department, Salman Bin Abdulaziz University, Riyadh, Saudi Arabia. \\ * Corresponding author. Tel.: +49-271-740-3480; email: rami.al-jarrah@uni-siegen.de \\ Manuscript submitted November 29, 2014; accepted June 14, 2015. \\ doi: 10.17706/ijcce.2016.5.3.215-221
}

\begin{abstract}
The aerial robots represent an interested and rich area of research because they are very useful to perform complex tasks such as localization and tracking targets. To develop blimp system that is appropriate in diversity scenarios, an intelligent control with high autonomy degree is required. Therefore, we design blimp robot based on embedded system; then, we present several fuzzy sets models that should deal with autonomous, navigation and visual tracking problems. These models are empirically designed by combining the possibilities distributions theory with fuzzy logic. Thus, this paper addresses the problem of tracking robots in parallel with achieving the cooperative behavior based on computer vision system and artificial intelligent control to improve the efficiency of such system. In addition, considering use of wireless sensor networks for estimation multi-targets locations and the distances between them is presented.
\end{abstract}

Key words: Aerial robot, fuzzy control, image processing, servoing visual control, wireless sensor networks.

\section{Introduction}

Recently, the cooperative of aerial-ground robot systems has become widely used in several applications such as target detection and tracking. In accordance with that, designing and developing an artificial intelligent team consists of cooperative autonomous aerial-ground robots with efficient performance has been one of the most challenging goals in robotic research. The cooperation of such system has involved state of problems such as target tracking. The main purpose of tracking system is simply regarding to the fact that the tracking can help and improve the ability of the aerial robot to cooperate with other robots in the system and share information. Perhaps the drones can explore the different terrains and areas. Unfortunately, their measures do not provide a high resolution for the exploration area since they fly fast at a quite high altitude [1]. However, none of the currently operational drone can reliably distinguish between legitimate military targets and civilian persons to avoid erroneous targeting [2]. Therefore, it becomes very essential to look for another solution which has more advantages over drones. The airship robot is one of the most important solutions in this field. It has several advantages such as long time hovering, take off and land vertically without runways , much less energy consumed, and cost efficiency [3]-[6]. Nevertheless all of these advantages, but in order to build an artificial blimp robot system, an eye (camera) is also important for this kind of robot. It can provide a high artificial visual system that simulates the principle and characteristics of the human eye system. Thus, if the robot can measure the range by only single vision sensor that could help reduces the overall cost of the robot. The computer vision community has proposed 
various methods to stabilize camera motions by tracking features [7]-[9] or by computing optical flow [10]-[15]. The common process in vision system is called a visual tracking that is analyzing of sequential images to identify a reference pattern and follow a moving interest point or defined object over time on the image. There are many tracking methods [16]-[20], but they could not meet the requirements of real time and robustness due to large amount of video data. Therefore, in order to improve the detection of targets in real time and robustness, the Speeded Up Robust Features (SURF) has been proposed [21]. Since the localization issue is the most important problem in the aerial robots and it is inevitable challenge and unsolvable problem, several applications on the localization are addressed [22]-[25]. However, one successful solution for the localization is based on the use of Wireless Sensor Networks (WSNs). We consider a 3D multi target localization problem where targets are flying within the range of the sensor network. Moreover, sensors modulate their decisions using On-Off Keying (OOK) which has been proposed recently for WSN because it is a power efficient modulation technique and enables sensors to use censoring scheme (send/no send).

\section{Problem Description and Methods}

The problem can be decomposed into four sub-problems: blimp localization, target tracking, cooperation, and finally the localization and tracking the whole system based on WSNs. The algorithm focuses on the single-blimp-target problem so that the blimp performs robust target tracking. Actually, there are four main important factors in order to design robust and reliable tracking for such system. First, the complexity of the environment. Second, the prior knowledge of target motion which might be random or unpredictable. Third, the targets we attempt to track are any complex and moving objects. Finally, based on the current blimp pose and the current target position, the blimp controllers must cope with these issues and provide the blimp an ability to move toward the target.

\subsection{Feature Descriptors and Tracking}

Feature description defines as computing process of the interest points in the image. There are several feature descriptors suitable for visual matching and tracking. The most widely used descriptors are Scale Invariant Feature Transform (SIFT) and Speeded UP Robust Feature algorithm (SURF) [26], [27]. Since the processing time of SURF algorithm is faster than that of SIFT, interest point detection of SURF algorithm is could be used for real time processing. Fig. 1 shows the results of SURF feature points matching. The perspective effect must be taken into account when processing images in order to weight each pixel according to its information content. In order to deal with such problem the Inverse Perspective Mapping (IPM) has been introduced [28]. It can help to remove the perspective effect from the acquired image, remapping it into a new 2D domain as it is shown in Fig. 2.

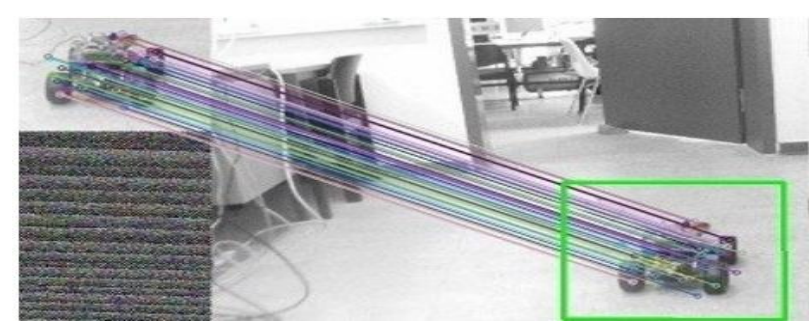

Fig. 1. SURF feature points matching.

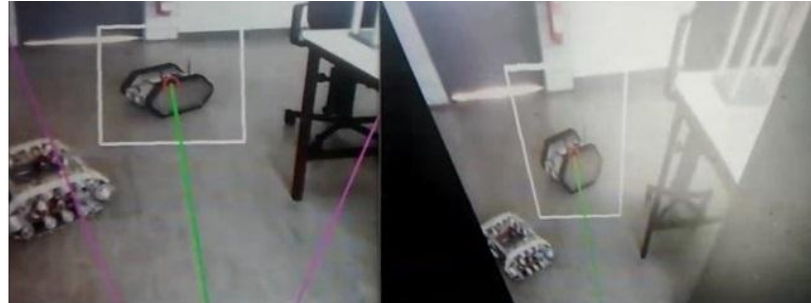

Fig. 2. Inverse perspective mapping results.

\section{Visual Servoing Controller}

The visual references on the image plan to directly command the blimp robot to continuously follow 
aerial or ground robot target. These controllers use the principles of visual servoing theory. The visual servoing was divided into four main parts: the SURF algorithm. The fuzzy sets models, low level control inputs and the visual control as it is shown in Fig. 3.

\subsection{Fuzzy Sets Models}

In fact, we have designed multi fuzzy sets models to perform the navigation tasks and to enable the blimp's system to autonomously drive the necessary actions to fulfill its mission and to maintain its policy during the navigation time. The main object in designing these models was to solve the most important problems with fuzzy logic which is how to design the fuzzy knowledge base or the membership functions. Therefore, the combination between the possibility theory and frequency distribution theory leads to design the fuzzy sets models. These models will correct the information and reduce the drawbacks of the mounted sensors such as ultrasonic sensors and vision sensor. The experimental results in Fig. 4 indicates that the fuzzy set model could correct the prediction visual position information for the SURF algorithm. Also, the model is pretty good in optimization when the value of prediction is outliers and has no sense.

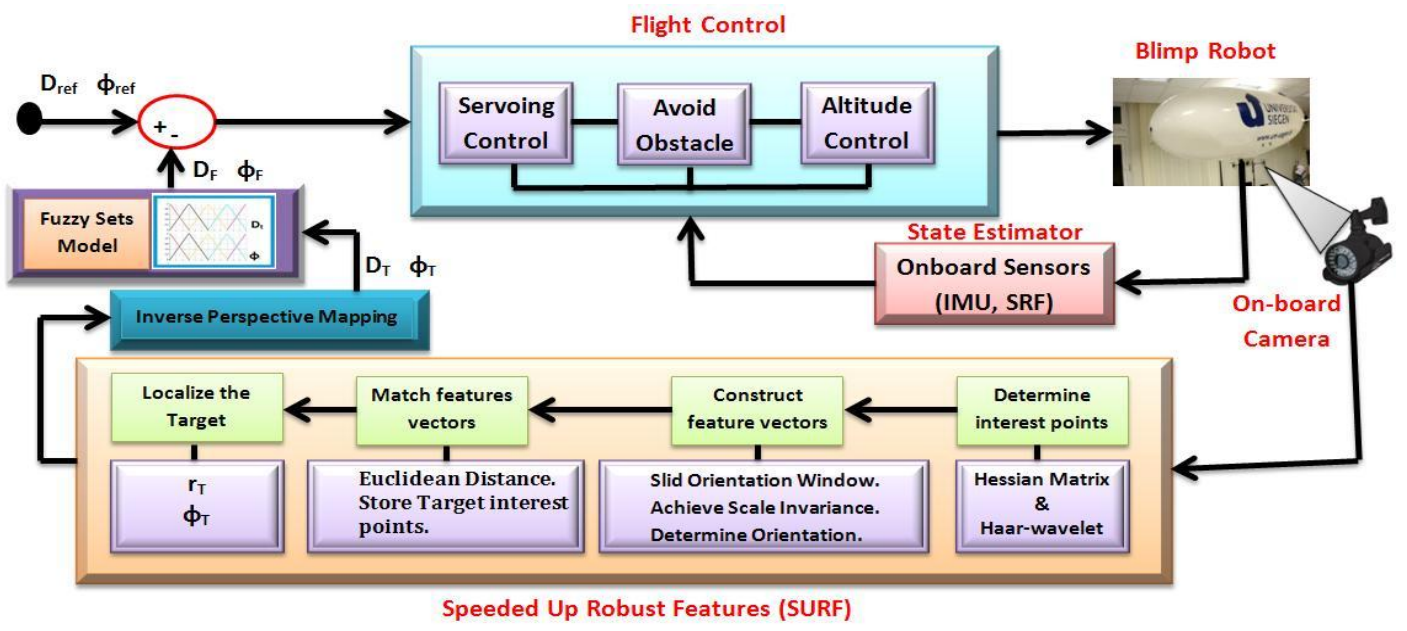

Fig. 3. Overall blimp control system architecture.

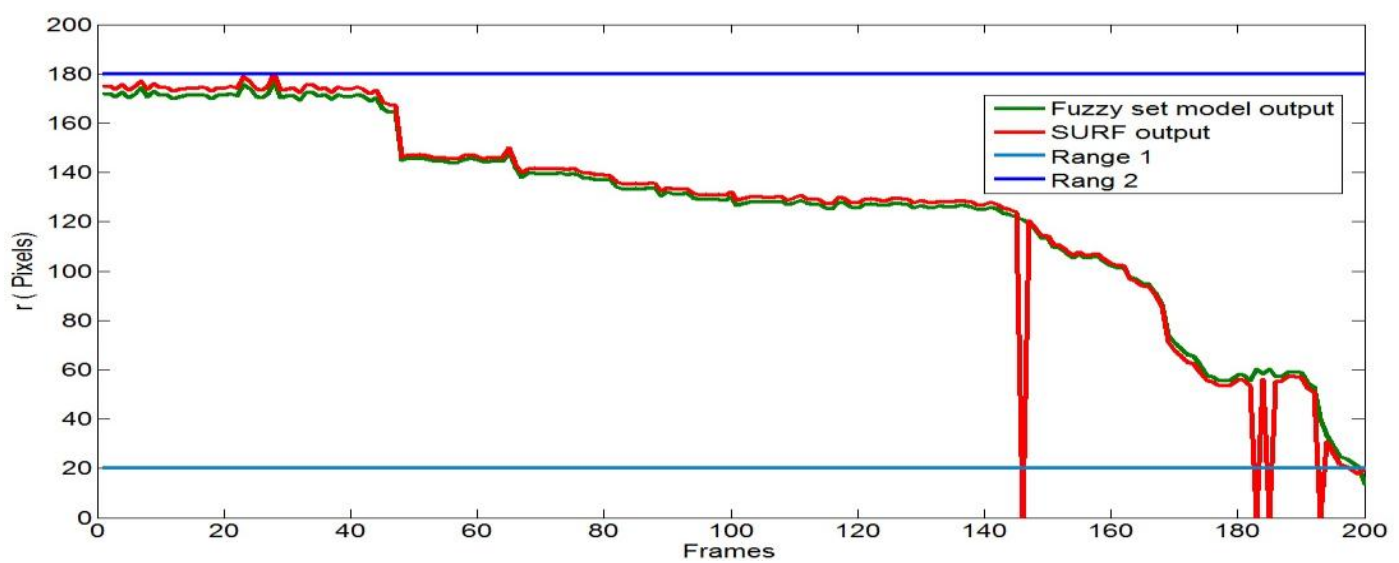

Fig. 4. Test results for fuzzy set model for different distances.

\subsection{Fuzzy Control}

There are three fuzzy controllers in order to control the main behaviors of the blimp robot during the mission. First, the most important behavior for the blimp robot is the altitude that is responsible to keep the blimp flies at a certain height. Second important behavior of the blimp that could be consider during the 
design is the avoid obstacles during mission. Since the blimp robot is stable around $\mathrm{x}$, and $\mathrm{y}$ axis it was better to control the rotation around Z-axis to keep the blimp stable. This was done by control the yaw angle by introducing intelligent control based on fuzzy logic [29]-[32]. The control strategy is based on the visual information to make the blimp complete the whole navigation performance and approaching the target. The vision feedback was optimized also by the fuzzy sets model to correct the prediction position information as it was described, then it is integrated with the blimp's flight control system to guide it to follow the target robot. The object recognition, detection and tracking engine will detect and localize the target with respect to blimp position; then a fuzzy sets model will correct the prediction vision information (distance to the target $\mathbf{D}_{\mathbf{t}}$, orientation of the target $\boldsymbol{\Phi}$ ) to obtain the final information (optimized distance $\mathbf{D}_{\mathbf{f}}$, optimize orientation $\boldsymbol{\Phi}_{\mathrm{f}}$ ) that will feedback the blimp .

\section{Wireless Sensor Networks Tracking}

The WSNs consists of a large number of low-cost and low-power sensors, which are deployed in an environment to collect observations and preprocess them in order to obtain local decisions and to detect ground and aerial targets including our blimp system if they pass through this region. The maximum likelihood estimator was used to estimate the location of the target where each sensor sends a row of quantized decisions to the fusion center [33], [34]. Depending on all rows sent by different sensors, the fusion center use maximum likelihood estimator to estimate the location of the target. Moreover, the efficiency of maximum likelihood estimator is compared with the weighted average algorithm and the Cramer-Rao lower Bound (CRLB). Moreover, sensors modulate their decisions using on-off keying OOK technique which has been proposed recently for WSNs because it is a power efficient modulation technique and enables sensors to use censoring scheme (send/no send). The mean square error of the Cartesian coordinates of each target location for different number of sensors has been calculated and compared to the CRLB. To maximize the maximum likelihood function, particle swarm optimization (PSO) method is used [34].

\section{Experiments and Results}

In this section, the results of design blimp robot, computer vision approaches, intelligent fuzzy sets model fuzzy controllers as well as the multi-target tracking results are discussed. The experiments are performed to test the capability of the approaches that have been described in the previous sections. The stability issues of visual control has been studied well and could be considered as solved in this paper.

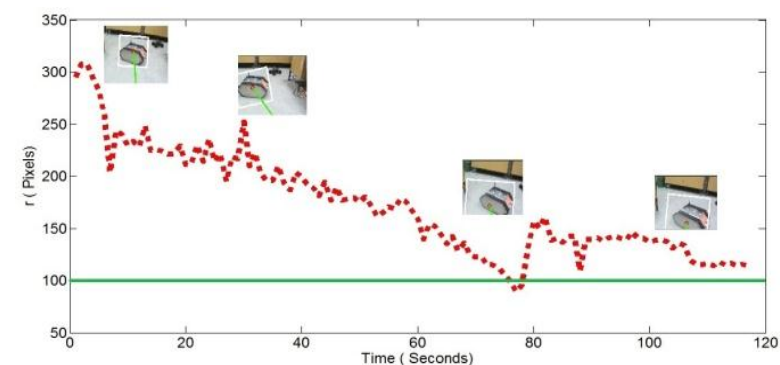

Fig. 5. Distance between target and blimp.

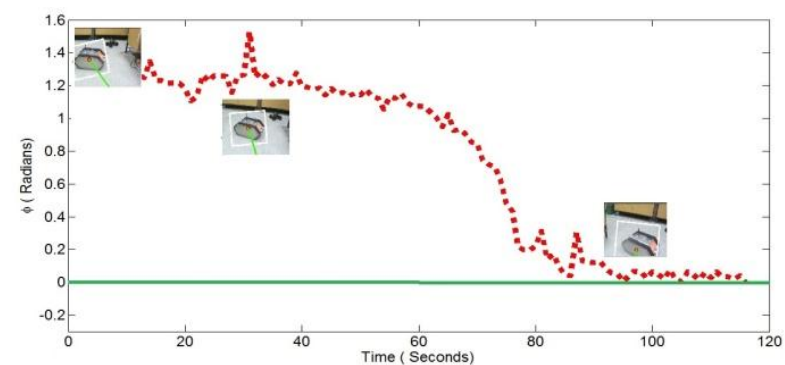

Fig. 6. Angle target, blimp and image’s centre.

Fig. 5 shows the estimated distances between target projection point and blimp. We observe that blimp can track target and keep it at a certain distance. The estimated angle between target projection point and image's centre results are shown in Fig. 6. In order to localize the blimp and the tracking targets WSNs has been used. Since it is not possible to estimate target height with single sensor due to observability problem, we combined the information from two or more sensors. In order to study the effect of number of sensors 
and minimize the number to localize the robots targets in the environment, the number of sensors was changed from 5 to 17 sensors as it is shown in Fig. 7 and Fig. 8. Whilst the number of sensors increased, the estimated locations are increased.

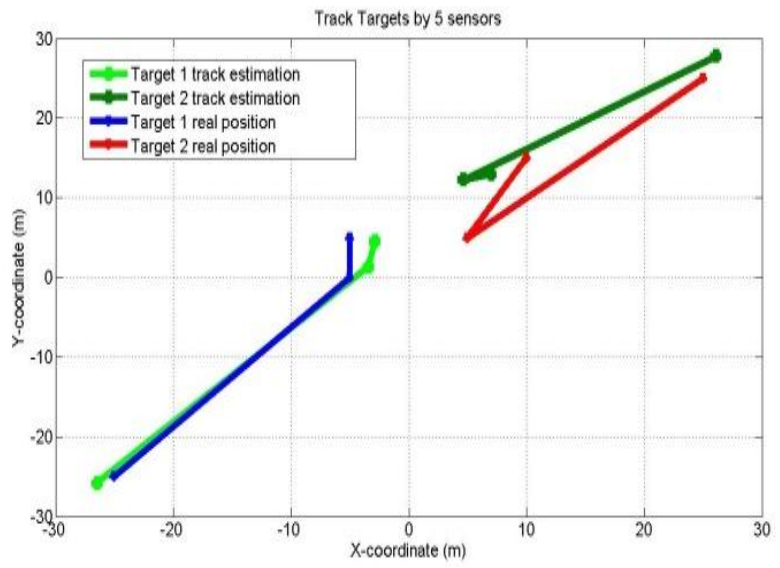

Fig. 7. Track two targets by 5 sensors.

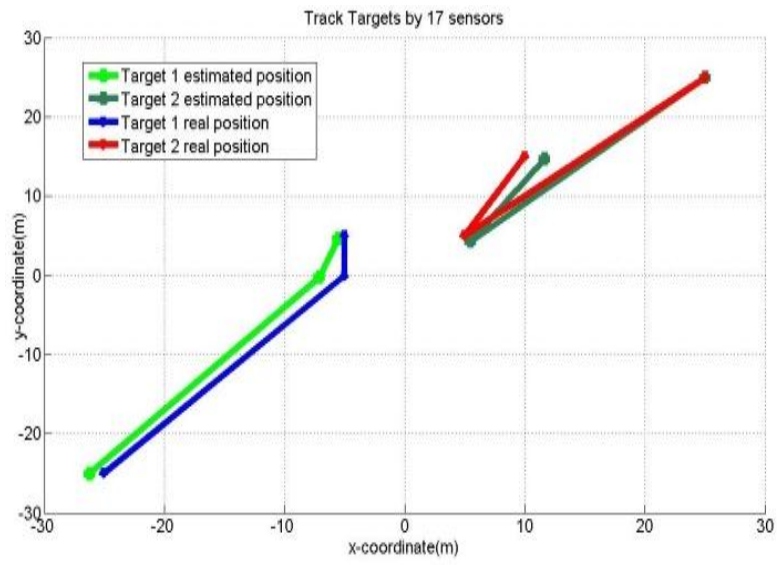

Fig. 8. Track two targets by 17 sensors.

\section{Conclusion}

We presented a solution for single robot-based tracking problem using aerial robot (Blimp Robot). For this purpose, we presented the tracking, localization and object detection method using SURF algorithm, then, the fuzzy sets models was implemented to handle the noise and uncertainty of the vision sensor. The IPM is also implemented in order to transform the images sequences to a top down bird's eye view, in which there is a linear relationship between distances in the image and in the real world. Then, this tracking method is used to estimate a projection model in the camera image space. The proposed method has been implemented and tested in various indoor environments.

\section{References}

[1] Xu, T., Zhang, X., \& Lu, Y. (2011). Onboard controlling system design of unmanned airship. Proceedings of International Conference on Electronic \& Mechanical Engineering and Information Technology: Vol. 6 (pp. 3028-3031).

[2] Melzer, N. (2013). Human rights implications of the usage of drones and unmanned robots in warefare. European Union, Belgium.

[3] Elfes, A., Bueno, A., \& Bergerman, M. (2003). Robotic airships for exploration of planetary bodies with an atmosphere: Autonomy challenges. Autonomous Robots Journal, 14, 147-164.

[4] Kitt, W. W., Chekima, A., Dhargam, J., Wong, F., \& Tabet, T. A. (2010). Soft computing control system of an unmanned airship. Iraq Electrical and Electronic Engineering Journal, 1, 22-27.

[5] Lin, Z.-J., \& Peng, X. D. (2009). Low altitude photogrammetry of unmanned airships. Proceedings of IEEE International Conference on Information Engineering and Computer Science (pp. 1-4).

[6] Moutinho, A., \& Azinheira, J. R. (2005). Stability and robustness analysis of the AURORA airship control system using dynamic inversion. Proceedings IEEE of International Conference on Robotics and Automation (pp. 2265-2270).

[7] Tomasi, C., \& Kanade, T. (1991). Detection and tracking of point features. Technical Report CMU-CS-91-132, Shape and Motion from Image Streams, Carnegie Mellon University, Pittsburgh, PA.

[8] Liu, T., Carlberg, M., Chen, G., Chen, J., Kua, J., \& Zakhor, A. (2010). Indoor localization and visualization using a Huma-operated backpack system. Proceedings of the IEEE International Conference on Indoor 
Positioning and Indoor Navigation (pp. 1-10).

[9] Liu, H., Chen, S., Zhang, Y., \& Chen, J. (2012). Modeling radar tracking features and low observability motion planning for UCAV. Proceedings of the IEEE 4th International Conference on Intelligent HumanMachine Systems and Cybernetics: Vol. 2 (pp. 162-166).

[10] Lucas, D. B., \& Kanade, T. (1981). An iterative image registration technique with an application to stereo vision. Proceedings of the Imaging Understanding Workshop (pp. 121-130).

[11] Cmko, P., \& Capson, D. (2012). Biomimetic measurement of optical flow and centroid for visual-servo control of hover flight. Proceedings of IEEE International Conference on Instrumentation and Measurement Technology (pp. 348-353).

[12] Denman, S., Fookes, C., \& Sridharam, S. (2009). Improved simultaneous computation of motion detection and optical flow for object. Proceedings of the IEEE International Conference on Digital Image Computing: Techniques and Applications (pp. 175-182).

[13] Chen, C. H., \& Chan, Y. P. (2007). Real time multi-target visual tracking based on velocity segmentation technique. Proceedings in IEEE of the 33rd Annual Conference of the IEEE Industrial Electronics Society (pp. 2813-2817).

[14] Huang, C. M., \& Hung, M. H. (2014). Target motion compensation with optical flow clustering during visual tracking. Proceedings of the IEEE of the 11th International Conference on Networking, Sensing and Control (pp. 96-101).

[15] Kumar, V., Rus, D., \& Sukhatme, G. S. (2008). Networked robots. Springer Handbook of Robotics, Part E: Networked Robots, 41, 943-958.

[16] Mikolajczyk, M., \& Smid, C. (2005). A performance evaluation of local descriptors. IEEE Trans. on Pattern Analysis and Machine Intelligence, 27(10), 1615-1630.

[17] Nister, D., Naroditsky, O., \& Bergen, J. (2006). Visual odometry for ground vehicle applications. Journal of field Robotics, 23(1), 3-20.

[18] Bonin-Font, F., Ortiz, A., \& Oliver, G. (2008). Visual navigation for mobile robots: A survey. Journal of Intelligent and Robotic Systems, 53, 263-296.

[19] Ishikawa, S., Kuwamoto, H., \& Ozawa, S. (2002). Visual navigation of an autonomous vehicle using white line recognition. IEEE Transactions on Pattern Analysis and Machine Intelligence, 10(5), 743-749.

[20] Matsumoto, Y., Inaba, M., \& Inoue, H. (1996). Visual navigation using view sequenced route representation. Proceedings of IEEE International Conference on Robotics and Automation: Vol. 1 (pp. 83-88).

[21] Bay, H., Ess, A., Tuyelaars, T., \& Gool, L. V. (2008). Speeded up robust features. Journal of Computer Vision and Image Understanding, 110(3), 346-359.

[22] Yim, J., Park, C., Joo, J., \& Jeong, S. (2008). Extended Kalman filter to wireless LAN based indoor positioning. Decision and Support Systems (Elsevier), 45, 960-971.

[23] Yim, J., Jeong, S., Gwon, K., \& Joo, J. (2010). Improvement of Kalman filters for WLAN based indoor tracking. Expert Systems Applications (Elsevier), 37(1), 426-433.

[24] Röhrig, C., \& Spieker, S. (2008). Tracking of transport vehicles for warehouse management using a wireless sensor network. Proceedings of IEEE/RSJ International Conference on Intelligent Robots and Systems, Nice, France, September 22-26.

[25] Röhrig, C., \& Muller, M. (2009). Localization of sensor nodes in a wireless sensor network using the nanoLOC TRX transceiver. Proceedings of IEEE 69th Vehicular Technology Conference Spring, Barcelona, Spain, April 26-29.

[26] Lowe, D. G. (1999). Object recognition from local scale-invariant features. Proceedings on IEEE of the 17th International Conference on Computer Vision: Vol. 2 (pp. 1150-1157). 
[27] Bay, H., Tuytelaars, T., \& Gool, L. V. (2006). SURF: Speeded up robust features. Processings of the Ninth European Conference on Computer Vision (pp. 404-417)

[28] Mallot, H. A., Bulthoff, H. H., Little, J. J., \& Bohrer, S. (1991). Inverse perspective mapping simplifies optical flow computation and obstacle detection. Biological Cybernetics, 64, 177-185.

[29] Al-Jarrah, R., \& Roth, H. (2014). Visual fuzzy control for blimp robot to follow 3D aerial object. Proceedings of 8th International Conference on Neural Network and Artificial Intelligence (pp. 98-111).

[30] Al-Jarrah, R., Ait-Jellal, R., \& Roth, H. (2013). Blimp based on embedded computer vision and fuzzy control for following ground vehicles. Proceedings of the 3rd IFAC Symposium on Telematics Applications, Seoul, Korea.

[31] Al-Jarrah, R. \& Roth, H. (2013). Design blimp robot based on embedded system \& software architecture with high level communication and fuzzy logic. Proceedings in IEEE of the 9th International Symposium on Mechatronics \& Its Applications (ISMA13), Amman, Jordan.

[32] Al-Jarrah, R., \& Roth, H. (2013). Developed blimp robot based on ultrasonic sensors using possibilities distributions and fuzzy logic. Journal of Automation and Control Engineering, 1(2).

[33] Al-Jarrah, M. A., Al-Ababneh, N. K., Al-Ibrahim, M. M., \& Al-Jarrah, R. (2014). Cooperative OFDM for semi distributed detection in wireless sensor networks. AEU-International Journal of Electronics and Communications, 68(10), 1022-1029.

[34] Al-Jarrah, R., Al-Jarrah, M. A. \& Roth, H. (2014). Multiple 3D target tracking in binary wireless sensor network. International Journal of Materials Science and Engineering, 3(1).

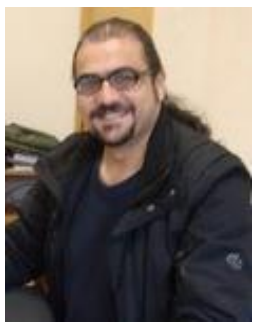

Rami Al-Jarrah is currently a PhD student and research assistant at Lehrstuhl für Regelungs, und Steuerungstechnik, Automatic Control Engineering Department (RST), Siegen University, Germany. His research interests are fuzzy logic analysis, image processing, wireless sensor networks and aerial robotics.

He worked as a lecturer in the Mechanical Engineering Department at Jordan University of Science \& Technology in 2002-2005 and in the Technical and Vocational Training Corporation, Computer Engineering Department, Saudi Arabia, 2006-2009.

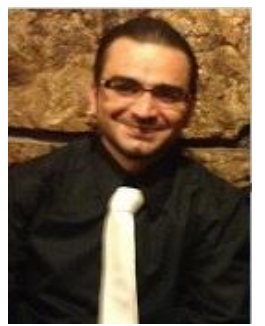

Mohammad A. Al-Jarrah received his master degree in electrical engineering (wireless communication engineering) from Jordan University of Science and Technology. He works now as a lecturer and researcher at the College of Engineering at Wadi Aldawaser, Department of Electrical Engineering at Salman Bin Abdulaziz University, Saudi Arabia.

His current area of research includes: distributed detection system, statistical signal processing, wireless sensor networks, cognitive radio networks, cooperative diversity, orthogonal frequency division multiplexing (OFDM), code division multiple access (CDMA) and fuzzy logic control.

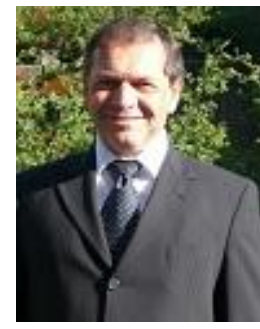

Hubert Roth was born in Germany, he completed his doctorate degree at Karlsruhe Technical University, Germany in 1983. Currently he is the chair in the Control Engineering Department (RST) at Siegen University, Faculty of Electrical Engineering and Informatics since 2001.

Prof. Dr.-Ing. Roth is a vice director of the Technical Committee on Computers and Telematics of International Federation of Automatic Control (IFAC) since 2008 and a vice director of Centre for International Capacity Development (CICD) since 2009. 